\title{
Rhabdomyosarcoma - an Epidemiological and Histopathologic Study of 277 Cases from a Major Tertiary Care Center in Karachi, Pakistan
}

\author{
Zubair Ahmad ${ }^{1}$, Nasir Ud Din ${ }^{1 *}$, Arsalan Ahmad ${ }^{1}$, Sheharbano Imran ${ }^{1}$, Shahid \\ Pervez $^{1}$, Rashida Ahmed ${ }^{1}$, Naila Kayani ${ }^{1}$
}

\begin{abstract}
Aim: To present the epidemiologic data (age, gender, size etc) and histopathologic and immunohistochemical features of cases of rhabdomyosarcoma (RMS) reported in our department. Settings: Section of Histopathology, Department of Pathology and Microbiology, Aga Khan University Hospital, Karachi, Pakistan. Duration: All cases of RMS diagnosed between January 1, 2003 and December 31, 2012 were included in the study. Results: A total of 277 cases were included. Embryonal rhabdomyosarcoma (ERMS) was by far the dominant histologic type $(87.4 \%)$ followed by alveolar type (ARMS)(9.4\%). ERMS was much more common in males (64.0\%)and over $65 \%$ of cases occurred in the first decade of life (over $90 \%$ in the first two decades). Head and neck region was commonest site for ERMS (46.7\%), followed by the genitourinary system (16.1\%). Over 65\% cases of ARMS occurred in the extremities. Over $80 \%$ cases of ARMS occurred in the first 3 decades of life. Immunohistochemical staining for Desmin and MyoD1 was positive in $96.7 \%$ and $85.4 \%$ respectively. Conclusions: The epidemiologic data and microscopic findings in our patients are similar to international published data on rhabdomyosarcoma.
\end{abstract}

Keywords: Rhabdomyosarcoma - embryonal type - alveolar type - pleomorphic type - epidemiology

Asian Pac J Cancer Prev, 16 (2), 757-760

\section{Introduction}

Rhabdomyosarcoma is the commonest soft tissue sarcoma in children and adolescents (Ognjanovic et al., 2009). Most cases occur in children under 10 years age and less than 20\% occur in adolescents(Gurney et al., 1996; Ognjanovic et al., 2009). Embryonal rhabdomyosarcoma (ERMS) is the most common histologic subtype comprising about $60 \%$ of all rhabdomyosarcomas(Sultan et al., 2009). ERMS is defined as a primitive, malignant soft tissue tumor with phenotypical and biological features of embryonic skeletal muscle cells (WHO, 2013). Embryonal RMS, botryoid type is a variant of ERMS with a characteristic gross (polypoid or grape-like) and histologic (aggregates of tumor cells tightly abutting an epithelial surface/cambium layer) appearance and comprises about $6 \%$ of all RMS (Newton et al., 1995; WHO, 2013). Alveolar RMS (ARMS) is the second most common subtype comprising of about $30 \%$ of all RMS, is more common in adolescents and young adults, unlike ERMS is uncommon in young children (under 10) and has a peak incidence between 10 and 25 years of age (Newton et al., 1995). By definition, it has a monomorphic population of primitive cells with round nuclei and features of arrested myogenesis(WHO, 2013). Other
RMS subtypes include pleomorphic rhabdomyosarcoma (PRMS, which mainly occurs in the sixth to seven decades of life (Stock et al., 2009); and spindle cell/sclerosing RMS which is an uncommon subtype comprising 5-10\% of all rhabdomyosarcomas (Chiles et al., 2004). Except for ARMS which shows no gender predilection, all other subtypes are more common in males (Parkin et al., 1988; Chiles et al., 2009; Ognjanovic et al., 2009; Stock et al., 2009). ERMS is much more common in whites, while ARMS does not show any racial or geographic predilection (Chiles et al., 2009; Ognjanovic et al., 2009). About half of ERMS occur in the head and neck and about half in the genitourinary tract, while ARMS most commonly occurs in the extremities (Harms, 1995; Raney et al., 2011).

The Intergroup Rhabdomyosarcoma Studies (IRS) group proposed a classification of rhabdomyosarcoma based on prognosis and divided RMS into three groups: superior prognosis (botryoid RMS and spindle cell RMS), intermediate prognosis (ERMS) and poor prognosis (ARMS and PRMS) (Newton et al., 1995). Age is an independent prognostic factor; children aged 1-9 years have a better prognosis. Stage and site of origin are also important prognostic factors (Meyer and Spunt., 2004; Oshi et al., 2004; Arndt et al., 2009). 
The aim of this study is to present epidemiologic data (age, gender, site) as well as pathologic findings (microscopic, immunohistochemistery) in a large series of patients diagnosed over a period of 10 years in the largest center for histopathology in Pakistan. To our knowledge, this is the largest series on Rhabdomyosarcoma from Pakistan, a populous country in South Asia with a population of nearly 190 million. Earlier, Bhurgri et al published data of rhabdomyosarcoma in Karachi, 19982002 (Bhurgri et al., 2004).We receive cases from the whole country through our collection points located all over Pakistan.

\section{Results}

A total of 277 cases of rhabdomyosarcoma were diagnosed during the period 2003 to 2012. Rhabdomyosarcoma comprised $13.8 \%$ of all soft tissue sarcomas diagnosed in our section during the study period. Embryonal rhabdomyosarcoma was the overwhelming predominant histologic type occupying for $87.4 \%$ of all cases. This was statistically significant (p-value:0.01). The breakup is shown in Table 1 .

Table 1. Break-down of Cases According to Histologic Type $(\mathbf{n}=277)$

\begin{tabular}{clrc}
\hline Serial no & \multicolumn{1}{c}{ Histologic Type } & Number & Percentage (\%) \\
\hline 1 & Embryonal (including & 242 & 87.4 \\
& Botryoid variant) & & \\
2 & Alveolar & 26 & 9.4 \\
3 & Plemorphic & 5 & 1.8 \\
4 & Rhabdomyosarcoma NOS & 4 & 1.4 \\
\hline
\end{tabular}

Table 2. Age-wise Distribution of ERMS (n=242)

\begin{tabular}{cccc}
\hline Serial no & Decade (years) & Number & Percentage (\%) \\
\hline 1 & $0-10$ & 159 & 65.7 \\
2 & $11-20$ yrs & 62 & 25.6 \\
3 & $21-30$ & 14 & 5.8 \\
4 & $>31$ & 7 & 2.5 \\
\hline
\end{tabular}

Table 3. Age-wise Distribution of ARMS (n=26)

\begin{tabular}{cccc}
\hline Serial no & Decade (years) & Number & Percentage $(\%)$ \\
\hline 1 & $0-10$ & 9 & 34.6 \\
2 & $11-20$ yrs & 72 & 6.9 \\
3 & $21-30$ & 5 & 19.2 \\
4 & $31-40$ & 2 & 7.7 \\
5 & $>40$ & 3 & 11.5 \\
\hline
\end{tabular}

Table 4. Break-down of ERMS Cases According to Location ( $\mathrm{n}=\mathbf{2 4 2})$

\begin{tabular}{clcc}
\hline Serial no & \multicolumn{1}{c}{ Site } & Number & Percentage (\%) \\
\hline 1 & Head and neck & 113 & 46.7 \\
2 & Genitourinary system & 39 & 16.1 \\
3 & Abdomen & 17 & 7 \\
4 & Pelvis & 8 & 3.3 \\
5 & Retroperitoneum & 2 & 0.8 \\
6 & Perineum/anal region & 9 & 3.7 \\
7 & Trunk & 9 & 3.7 \\
8 & Extremities & 24 & 9.9 \\
9 & Other sites* & 10 & 4.1 \\
10 & Site not known & 11 & 4.6 \\
\hline
\end{tabular}

*The other sites included: vertebrae/spine ( 5 cases), kidney ( 2 cases) and mediastinum ( 3 cases).
There were 26 cases of Botryoid variant comprising $10.7 \%$ of embryonal rhabdomyosarcomas and $9.4 \%$ of all rhabdomyosarcomas. Of the 242 cases of ERMS, $155(64.04 \%)$ were males and 87(35.96\%) were females. Male to female ratio was $1.78: 1$. The predominance of ERMS in males was statistically significant (p-value:0.006). Age range for ERMS was 4 months to 65 years with mean and median age of 9 and 8 years respectively. The decade wise breakup of ERMS cases is shown in Table 2.

As shown in Table 2, 65.7\% cases of ERMS occurred in the first decade of life. This finding was statistically significant (p-value:0.094). Out of the 242 cases of ERMS, $109(45.04 \%)$ occurred in patients 5 years of younger. This finding was statistically significant (p-value:0.001). The 26 cases of ARMS were evenly distributed between males and females i.e. 13 cases each. Age range was 2 to 74 years with mean and median age of 21 and 24 years respectively. The decade wise breakup of ARMS cases is shown in Table 3.

Head and neck was the commonest site of ERMS with $47.7 \%$. The breakup of ERMS according to location is given in Table 4.

The further break up of head and neck and genitourinary cases of ERMS is given in Table 5.

The majority of 26 cases of ARMS (65.4\%) were located in the extremities. The breakdown of 26 cases of

Table 5. Break-down of ERMS Head and Neck and Genitourinary Cases $(\mathbf{n}=152)$

\begin{tabular}{rlrr}
\hline Serial no & \multicolumn{1}{c}{ Location } & Number & Percentage(\%) \\
\hline & (A) Head and Neck & 113 & \\
1 & Nose \& Paranasal sinuses & 21 & 18.6 \\
2 & Nasopharynx & 9 & 8 \\
3 & Orbit/periorbital soft tissue & 23 & 20.3 \\
4 & Oral/oropharynx/cheek/palate & 20 & 17.7 \\
5 & Ear & 6 & 5.3 \\
6 & Tongue & 1 & 0.9 \\
7 & Conjunctiva & 1 & 0.9 \\
8 & Parotid & 1 & 0.9 \\
9 & Jaw & 14 & 12.4 \\
10 & Face & 2 & 1.8 \\
11 & Skull & 5 & 4.4 \\
12 & Neck & 5 & 4.4 \\
13 & Sphenoid & 4 & 3.5 \\
14 & Parameningeal & 1 & 0.9 \\
& (B) Genitourinary System & 39 & \\
1 & Urinary bladder & 22 & 56.4 \\
2 & Testicular/paratesticular soft tissue & 9 & 23.1 \\
3 & Prostate & 2 & 5.1 \\
4 & Vagina & 5 & 12.8 \\
5 & Cervix & 1 & 2.6 \\
\hline
\end{tabular}

Table 6. Breakup of Cases of ARMS According to Location $(n=26)$

\begin{tabular}{clcc}
\hline Serial no & \multicolumn{1}{c}{ Site } & Number & Percentage (\%) \\
\hline 1 & Extremities & 17 & 65.4 \\
2 & Head and Neck & 5 & 19.2 \\
& Neck & 2 & \\
& Nose & 1 & \\
& Nasopharynx & 1 & \\
3 & Retrobulbar & 1 & \\
4 & Trunk & 3 & 11.5 \\
& Abdomen & 1 & 3.8 \\
\hline
\end{tabular}


ARMS is given in Table 6.

Desmin was the main immunohistochemical(IHC) stain used to confirm the diagnosis. It was performed on 272 out of 277 cases. It was positive in overwhelming majority of cases i.e in 268 out of 272 cases $(96.7 \%)$ in which it was performed. We acquired the IHC stain MyoD1 in 2006 and started performing it together with Desmin in suspected cases of RMS. It was performed in 103 cases (out of 277 in this study), was positive in 88 out of 103 cases $(85.4 \%)$ and was negative in 15 cases (14.6\%). CD99 (MIC 2) was another marker which was commonly performed in these cases. It was positive in 29 cases $(19.3 \%)$ and negative in 121 cases $(80.7 \%)$. Smooth muscle actin (ASMA) was performed in 63 cases. It was positive in 27 cases $(42.8 \%)$ and negative in 36 cases $(57.2 \%)$. Other IHC markers performed in these cases (as routine workup of small round blue cell tumors) included LCA in 136 cases, negative in all, which was acquired in 2008 and performed in 12 cases, negative in all. Broad spectrum cytokeratins were performed in 118 cases and were negative in $112(94.9 \%)$ and positive in 6 cases $(5.1 \%)$.

Out of the total 277 cases included in the study, 8 were blocks received for second opinion. Of the remaining 269 cases, 117(43.5\%) were incisional biopsies, while 152 $(56.5 \%)$ were excisional biopsies/resection specimens.

\section{Discussion}

Rhabdomyosarcoma is the most common soft tissue sarcoma worldwide in children under 15 years of age and one of the commonest in adolescents and young adults. Studies from Asia and Africa (Missaoui et al., 2010; Liu et al., 2014) also endorse this. It comprises about $8 \%$ of all malignant tumors in children and 2 to $5 \%$ of all adults sarcomas (Gurney et al., 1997; Hawkins et al., 2001). Rhabdomyosarcoma comprised $13.8 \%$ of all soft tissue sarcomas diagnosed in our section during the study period. ERMS constituted the overwhelming majority $(87.4 \%)$ of cases in our study. This percentage is much higher than that reported in literature which is around $60 \%$ (Ognjanovic et al., 2009). Similarly, the Botryoid variant of ERMS was more common in our study $(9.4 \%$ of all cases) compared to previously published data i.e. around $6 \%$ (Newton et al., 1995). Owing to the overwhelming majority of ERMS in our study, the percentage of ARMS was correspondingly $(19.4 \%)$ which is much lower than that reported in literature which is about $31 \%$ (Newton et al., 1995).

In our study, there were no cases of sclerosing or epitheloid RMS which are rare or recently described entities (Chiles et al., 2004; Jo et al., 2011). It may be possible that few of the cases diagnosed as ERMS in our study in the earlier years may actually represent sclerosing variant of RMS since this variant occurs in both children and adults and mainly involves head and neck and paratesticular region (Cavazzana et al., 1992; Chiles et al., 2004). We may in future review all cases of RMS to detect any cases of sclerosing RMS in our series. We are yet to see a definite case of epithelioid RMS, a new entity which is not yet included in the WHO Classification
(Jo et al., 2011).

Botryoid RMS comprised $9.4 \%$ of all cases in our series, a figure slightly higher than the approximately $6 \%$ recorded in published series (Newton et al., 1995). This variant usually occurs in mucosa lined hollow organs like nasopharynx, nasal cavity, vagina, urinary bladder etc and enjoys an unrestricted growth in such hollow organs resulting in its characteristic grape like appearance (Newton et al., 1995). ERMS cases in our series were much more common in males (M:F ratio 1.7:1) Published data from US and Canada show similar figures (Parkin et al., 1988; Ognjanovic et al., 2009). As shown in Table 1 , almost $66 \%$ cases occurred in the first decade of life, while just over $45 \%$ occur in patients 5 years or younger. Western studies also show that most children are aged less than 10 years and about $36 \%$ occur under 5 years of life(Gurney et al., 1996; Ognjanovic et al., 2009).

ARMS cases were evenly distributed among males and females. Western literature also shows no gender predilection(WHO, 2013). As shown in Table 3, over $45 \%$ of ARMS occurred between the ages 11 to 30 years, while over $34 \%$ occurred in the first decade of life. International data shows that peak incidence is between 10 to 25 years of age with median age between 6.8 and 9.0 years (Caillaud et al., 1989).

As shown in Table 4, the most common location for ERMS in our series was head and neck (46.7\%) followed by genitourinary system $(16.1 \%)$. Western data shows that almost one half cases of ERMS occur in the head and neck region and one half occur in the genitourinary system, less than $9 \%$ occur in the extremities (Raney et al., 2011). In our study, almost $10 \%$ cases of ERMS occurred in the extremities. Within the head and neck, the commonest sites, as shown in Table 5 were paranasal sinuses and nasopharynx, orbit and periorbital soft tissues and oral cavity. Within the genitourinary system, the commonest sites for ERMS, as shown in Table 5, were urinary bladder followed by testis and paratesticular soft tissue. Head and neck and genitourinary system were also the commonest sites for cases of botryoid RMS in our series. International data also show these are the commonest locations for ERMS (Newton et al., 1995). As shown in Table 4, a number of cases in our study occurred in the abdomen, pelvis, retroperitoneum, perineum etc and 2 cases were seen in kidney. Western data also shows these sites to be common locations for ERMS. ERMS also occur in viscera like kidney and heart (WHO, 2011; Arndt et al., 2009). Similarly, a number of our cases occurred in the soft tissues of the trunk. Western data also shows similar features (WHO, 2011).

In our study, over $65 \%$ cases of ERMS were located in the extremities, while head and neck was also a relatively common site (Table). Western data also shows that ARMS occurs most commonly in the extremities while head and neck is another common location (Harms, 1995; Yasuda, 2009).

Desmin was the main IHC stain used to confirm the diagnosis and showed a high degree of sensitivity (positive in $96.7 \%$ cases). It is however, not as specific as myoglobin or myogenin, being positive in both striated and smooth muscle (Molenaar, 1985). We started using Myo D1 in 


\section{Zubair Ahmad et al}

2006 and since then it is regularly performed in suspected RMS cases. As shown in our results, it also showed a high degree of sensitivity (over 85\%). However, we have only recently acquired myogenin and this antibody plays an important role in the diagnosis of RMS cases. Studies have shown that these IHC stains (Myo D1 and myogenin) are highly specific and sensitive (only nuclear staining is specific) and are currently favoured for the confirmation of a diagnosis of RMS (Cessna, 2001; Morotti, 2006).

\section{References}

Arndt CA, Stoner JA, Hawkins DS, et al (2009). Vincristine, Actinomycin and cyclophosphamide alternating with vincristine, topotecan and cyclophosphamide for intermediate risk rhabdomyosarcoma: children's oncology group study D9803. J ClinOncol, 27, 5182-8.

Bhurgri Y, Bhurgri A, Puri R, et al (2004). Rhabdomyosarcoma in Karachi 1998-2002. Asian Pac J Cancer Prev, 5, 284-90.

Caillaud JM, et al (1989). Histopathologic classification of childhood rhabdomyosarcoma: a report from the international Society of Pediatric Oncology pathology panel. Med Pediatr Oncol, 17, 391-400.

Cavazzana AO, et al (1992). Spindle cell rhabdomyosarcoma. A prognostically favourable variant of rhabdomyosarcoma. Am J Surg Pathol, 16, 229-35.

Cessna MH, et al (2011). Are myogenin and Myo D1 expression specific for rhabdomyosarcoma? A study of 150 cases, with emphasis on spindle cell mimics. Am J Surg Pathol, 25, 1150-7.

Chiles MC, Parhaw DM, Qualman SJ, et al (2004). Sclerosing rhabdomyosarcoma in children and adolescents: a clinicopathologic review of 13 cases from the intergroup rhabdomyosarcoma study group and children's oncology group. Pediatr Dev Pathol, 7, 583-94.

Gurney JG, Davis S, Severson RK, et al (1996). Trends in cancer incidence among children in the U.S. Cancer, 78, 532-41.

Gurney JG, Ross JA, Wall DA, et al (1997). Infant cancer in the U.S. histology specific incidence and trends, 1973 to 1992. J Pediatr Hematol Oncol, 19, 428-32.

Harms D (1995). Alveolar rhabdomyosarcoma: a prognostically unfavourable rhabdomyosarcoma type and its necessary distinction from embryonal rhabdomyosarcoma. Curr Top Pathol, 89, 273-96.

Hawkins WG, Hoos A, Antonescu CR, et al (2001). Clinicopathologic analysis of patients with adult rhabdomyosarcoma. Cancer, 91, 794-803.

Jo VY, Marino-Enriquez A, Flethcher CD (2011). Epithelioid rhabdomyosarcoma; clinicopathologic analysis of 16 cases of a morphologically distinct variant of rhabdomyosarcoma. Am J Surg Pathol, 35, 1523-30.

Joshi D anderson JR, et al (2004). Age is an independent prognostic factor in rhabdomyosarcoma: a report from the soft tissue sarcoma committee of the children's oncology group. Pediatr Blood Cancer, 42, 64-73.

Liu CX, et al (2014). Compound HRAS/PIK3CA mutations in Chinese patients with alveolar rhabdomyosarcoma. Asian Pac J Cancer Prev, 15, 1771-4.

Meyer WH, Spunt SL (2004). Soft tissue sarcomas of childhood. Cancer Treat Rev, 30, 269-80.

Missaoni N, et al (2010). Pediatric rhabdomyosarcoma in Tunisia. Asian Pac J Cancer Prev, 11, 1325-7.

Molenaar WM, Oosterhuis JW, Oosterhuis AM, Ramaekers FC (1985). Mesenchymal and muscle specific intermediate filaments (vimentin and desmin) in relation to differentiation in childhood rhabdomyosarcoma. Hum Pathol, 16, 838-43. Morotti RA, et al (2006). An immunhistochemical algorithm to facilitate diagnosis and subtyping of rhabdomyosarcoma; the children's oncology group experience. Am J Surg Pathol, 30, 962-8.

Newton Jr WA, et al (1995). Classification of rhabdomyosarcomas and related sarcomas. Prognostic aspects and proposal for a new classification-an intergroup rhabdomyosarcoma study. Cancer, 76, 1073-85.

Ognjanovic S, Linabery AM, Charbonneau B, Ross JA (2009). Trends in childhood rhabdomyosarcoma incidence and survival in the United States, 1975-2005. Cancer, 115, 4218-26.

Parkin DM, Stiller CA, Drager GJ, Bieber CA (1988).The international incidence of childhood cancer. Int $J$ Cancer, 42, 511-20.

Raney RB, Walterhouse DO, Meza JL, et al (2011). Results of the intergroup rhabdomyosarcoma study group D9602 protocol, using vincristine and dactinomysin with or without cyclophosphamide and radiation therapy, for newly diagnosed patients with low risk embryonal rhabdomyosarcoma: a report from the soft tissue sarcoma committee of the children's oncology group. J Clin Oncol, 29, 1312-8.

Stock N, et al (2009). Adult-type rhabdomyosarcoma:analysis of 57 cases with clinicopathologic description, identification of 3 morphologic patterns and prognosis. Am J Surg Pathol, 33, 1850-9.

Sultan I, Qaddonmi I, Yaser S, Rodrigue-Galindo C, Ferrari A (2009). Comparing adult and pediatricrhabdomyosarcoma in the surveillance, epidemiology and end results program, 1973 to 2005: an analysis of 2600 patients. J Clin Oncol, 27, 3391-7.

WHO Classification of Tumors of Soft Tissue and Bone (2013). Fletcher CDM, Bridge JA, Hogendoorn PCW, Mertens Feds. $4^{\text {th }}$ Edition. International Agency for Research on Cancer (IARC), Lyon, 123-36.

Yasuda T, et al (2009). Alveolar rhabdomyosarcoma of the head and neck region in older adults: genetic characterization and review of the literature. Human Pathol, 40, 341-8. 